\title{
Application of a Multiscale Particle Scheme to High Altitude Rocket Exhaust Flows
}

\author{
Jonathan M. Burt ${ }^{1}$ and Iain D. Boyd ${ }^{2}$ \\ Department of Aerospace Engineering \\ University of Michigan, Ann Arbor, MI 48109
}

\begin{abstract}
A hybrid particle scheme for simulation of compressible gas flows involving a wide range of characteristic length scales and Knudsen number $(\mathrm{Kn})$ regimes is extended for application to gas mixtures. The direct simulation Monte Carlo (DSMC) method is utilized in high Kn regions where continuum flow simulation techniques are inaccurate, and a recently developed low diffusion (LD) particle method based on DSMC is employed in low Kn regions where DSMC is prohibitively expensive. A series of expressions are presented for determination of transport coefficients in a mixture, and a new model for diffusive mass transport in the LD method is proposed. This mass diffusion model is evaluated through a comparison with other numerical results for a one dimensional self diffusion test case, and good overall agreement is observed. To demonstrate capabilities of the newly modified LDDSMC algorithm, a large scale hybrid LD-DSMC simulation is performed for the exhaust and atmospheric flow around a generic sounding rocket at an altitude of $100 \mathrm{~km}$. The influence of mass diffusion is investigated, and an additional simulation is carried out for a similar flow involving stage separation.
\end{abstract}

\section{Introduction}

$\mathrm{D}$ ESPITE considerable progress over the past several decades, accurate and efficient simulation of rocket exhaust flows at high altitude remains a considerable challenge. This is due in part to numerical difficulties associated with the wide range of flow regimes encountered. For characterization of the full domain of interest in high altitude plume analysis, the internal flow within the combustion chamber and nozzle must be included, in addition to nearfield and farfield plume regions and the atmospheric flow region upstream of the nozzle. Strong translational nonequilibrium phenomena tend to occur in atmospheric and farfield plume regions at high altitudes (above roughly $100 \mathrm{~km}$ ) and require kinetic theory based simulation techniques, most commonly the direct simulation Monte Carlo (DSMC) method, ${ }^{1}$ which allow for arbitrary nonequilibrium gas velocity distributions. Such methods become prohibitively expensive, however, when applied to high density regions in the nozzle and nearfield plume where the local Knudsen number is very small. The Knudsen number $(\mathrm{Kn})$ is defined as the ratio of the mean free path to some characteristic length scale based on boundary geometry or flowfield structures, and is generally a good indicator of the degree of thermal nonequilibrium in a gas flow. In lower Kn regions where DSMC becomes intractable due to severe restrictions on computational cell size and time step, continuum computational fluid dynamics (CFD) methods which assume a near-equilibrium velocity distribution are both accurate and far more efficient.

\section{A. Uncoupled CFD and DSMC Simulations}

Due to the inefficiency of DSMC in low-Kn regions and the inaccuracy of CFD methods in high-Kn nonequilibrium regions, the typical procedure for high altitude rocket exhaust plume simulation involves a two step process. First, a simulation is run for flow within the nozzle and any high density nearfield plume regions, using a continuum CFD solver for the Navier-Stokes equations. A continuum breakdown surface is defined within the domain of this simulation, and is then used as an inflow boundary for an uncoupled DSMC simulation of the farfield plume and atmospheric flow regions. ${ }^{2-6}$ While this uncoupled technique does permit accurate simulation of most high altitude plume flow problems of practical interest, it has several limitations that lead to some desire for a simpler, faster and more general approach.

\footnotetext{
${ }^{1}$ Post-doctoral research fellow, AIAA member.

${ }^{2}$ Professor, AIAA associate fellow.
} 
One major disadvantage of the two step uncoupled simulation approach is the "man in the loop" issue; significant time may be required to set up two independent simulations, and to manually assign DSMC inflow properties along the continuum breakdown surface based on CFD simulation results. ${ }^{3}$ The presence of two overlapping simulation domains is a further inconvenience for data visualization, and may require the use of postprocessing algorithms to merge or interpolate values between multiple simulation output zones.

An additional disadvantage of the two step approach is its restriction to relatively simple flow configurations. As no information (i.e. characteristic lines) can travel from the DSMC domain into the CFD domain, the flow across the continuum breakdown surface must be uniformly supersonic in the surface-normal direction. While this condition is typically met for simple plume flowfield geometries, there are a number of cases where the flow at the breakdown surface is subsonic in the surface-normal direction, and where two-way coupling of flowfield information exists between continuum and rarefied regions. This limitation may be particularly problematic for plume impingent flows related to divert jet firings or rocket staging events. A further limitation of the uncoupled two step technique is a general assumption of steady state conditions. Because the location and flow properties along the DSMC inflow boundary are usually fixed, unsteady flows cannot be simulated without multiple simulations and considerable user effort.

\section{B. The Hybrid CFD-DSMC Approach}

One alternative to the standard two step simulation approach is a CFD-DSMC hybrid scheme, which involves two-way coupled DSMC and Navier-Stokes CFD calculations within a single numerical framework. ${ }^{7-13}$ This potentially allows for accurate characterization of the entire nozzle and plume flowfield in one simulation, using a single set of input parameters. Coupled CFD-DSMC hybrid schemes have been a subject of much research in recent years, and can at least partially resolve most of the problems inherent in the uncoupled two step approach. However, the use of CFD-DSMC hybrid algorithms introduces additional difficulties, particularly in the requirement for twoway information transfer along continuum breakdown boundaries. Coupling between CFD and DSMC simulation procedures - particularly the strong two-way coupling which may be needed for simulating unsteady flows requires special consideration of DSMC statistical scatter to avoid unphysical oscillations or instabilities in CFD calculations. Techniques for accurate coupling are generally complex, and are active topics of current research.

As an additional drawback to this type of approach, the integration of two entirely different simulation methods into a single code is both difficult and time consuming. It is therefore not surprising that no application of a coupled CFD-DSMC hybrid scheme to full scale high altitude rocket exhaust flows could be found in an extensive literature search. To date, most published applications of coupled hybrid schemes have focused on either hypersonic atmospheric flows or simplified model problems with few similarities to rocket exhaust flows.

\section{An Alternate Hybrid Approach}

One promising alternative approach for high altitude plume simulation is a hybrid particle scheme recently developed by the authors. ${ }^{14-16}$ As with hybrid CFD-DSMC algorithms, this scheme allows for simulations involving a wide range of Kn regimes - from continuum to free molecular - and allows two-way coupling between continuum and rarefied regions. However, in contrast to CFD-DSMC methods, implementation of the scheme in an existing DSMC code is relatively simple and straightforward, with an addition of only about $15 \%$ to the size of the DSMC source code. In the hybrid particle scheme, a continuum breakdown parameter (or a series of parameters) is periodically evaluated in all cells within a computational grid, and a smoothing technique is used with breakdown parameter evaluations to assign flowfield regions to either rarefied or continuum domains. DSMC calculations are performed for all cells within the rarefied domain, while a newly developed low diffusion (LD) particle method is used for cells within continuum regions.

The LD particle method is closely based on DSMC, and uses a series of near-equilibrium kinetic theory approximations to model molecular motion over length and time scales much larger than the mean free path and mean collision time respectively. ${ }^{14}$ Extensive evaluations of the LD particle method have been performed for boundary layer flows, shock tube flows and a two dimensional flow problem involving complex shock interactions. A high level of overall accuracy has been demonstrated for all of these cases. Due to relaxed cell size and time step requirements, as well as a low level of statistical scatter, the method has also been found to be far less expensive than DSMC or other DSMC-based methods intended for low Knudsen number flows. Unlike existing DSMC-based equilibrium particle methods, the LD particle method gives numerical transport coefficients which are only weakly dependent on cell size. ${ }^{14}$ As a result, low Knudsen number boundary layers, free shear layers and other high gradient regions can be accurately simulated without requiring prohibitively small cells and time step intervals.

A hybrid scheme involving both the LD method and DSMC has been recently presented, and the reader is referred to previous papers for details on implementation of the LD method and the hybrid algorithm. ${ }^{15,16}$ Hybrid 
LD-DSMC simulations have been performed for a hypersonic blunt body flow and a small scale nozzle and rarefied plume flow, both involving a simple (one species) gas. In both cases, excellent agreement with other available numerical and experimental data has been observed.

In the current effort, the hybrid LD-DSMC particle scheme is extended for simulating gas mixtures, and the modified scheme is applied to more complex, larger scale flows which represent typical configurations and conditions for a high altitude liquid propellant exhaust plume. While no existing data is available for comparison, these cases provide a demonstration of the capabilities of the hybrid scheme, and likely constitute the first effort at large scale unified nozzle/plume/atmospheric flow simulation with strong two-way coupling between continuum and rarefied regions.

\section{Outline of the Paper}

This paper is organized as follows: First, a general overview is provided for numerical procedures in the hybrid LD-DSMC scheme, and expressions are introduced for determining transport coefficients in LD method calculations involving a gas mixture. Next, small modifications to LD method procedures are described for inclusion of mass diffusion effects, so that species concentration gradients are influenced by diffusive transport at physically appropriate rates. A simple one dimensional mass diffusion problem is used to evaluate these new procedures, and LD simulation results are compared with a finite difference solution as well as DSMC results for the same problem. Results are then presented for hybrid LD-DSMC simulations of representative high altitude rocket exhaust cases. These cases include a unified simulation of nozzle, plume and atmospheric flow regions for an upper stage rocket operating at an altitude of $100 \mathrm{~km}$, and a modified version of this problem involving a stage separation event. Finally, results are summarized and ideas for future work are presented.

\section{Simulation Procedures in the Hybrid LD-DSMC Scheme}

As described in the introduction, the hybrid LD-DSMC scheme utilizes standard DSMC in high-Kn flowfield regions where DSMC is reasonably efficient, and uses a DSMC-based low diffusion (LD) particle method in low-Kn continuum regions where DSMC is prohibitively expensive due to cell size and time step limitations. Information is transported between LD and DSMC domains during each simulation time step through particle movement, and many procedures - particle movement, cell-based sampling, etc. - are nearly identical in the LD method as in DSMC. While the reader is referred to previous papers ${ }^{14-16}$ for details on the LD method and hybrid LD-DSMC simulation procedures, a brief outline of these procedures is provided here for reference.

\section{A. The Low Diffusion Particle Method}

The main difference between the LD method and DSMC is the set of procedures used to update particle properties during each time step. In DSMC these update procedures are carried out by selecting particles to participate in binary collisions, while in the LD method an alternate set of calculations are performed: First, cellbased quantities, such as bulk velocity and temperature, are computed for each cell by averaging among all particles currently assigned to the cell. These cell quantities are determined as functions of particle temperature and bulk velocity values, which are included in the particle data structure along with other values (such as a position vector and a separate velocity for particle movement) also used in DSMC.

Following determination of cell-based quantities, the velocity $u_{f}$ in the outward normal direction is calculated for a moving Lagrangian cell face corresponding to each cell face in the fixed Eulerian grid. Here Lagrangian cells are coincident with Eulerian cells at the beginning of each time step, but move and deform over the time step interval according to the bulk motion of the gas. Lagrangian faces are modeled as massless specularly reflecting wall boundaries, and $u_{f}$ values are determined through a momentum balance which involves approximating cells on either side of the face as semi-infinite reservoirs in local thermodynamic equilibrium. ${ }^{14}$ Once $u_{f}$ values have been found for all cell faces during the current time step in an LD method calculation, cell-based macroscopic flow properties (bulk velocity and temperature) are updated to account for momentum and energy transport across Lagrangian faces over the time step interval. Additional modifications to these cell-based quantities are performed to account for viscous transport phenomena, through a finite volume discretization of viscous terms in the compressible Navier-Stokes equations for a simple gas. ${ }^{16}$ Transport coefficients in these equations are based on the variable hard sphere (VHS) collision model, ${ }^{1}$ also assuming a simple gas. Resulting cell-based properties are then used to update the bulk velocity and temperature values for all particles assigned to the cell.

As a final stage in procedures for modifying particle properties during each time step, velocities used for particle movement are updated. New particle velocities are determined by finding the difference between final and initial particle positions over this time step, then dividing this difference by the time step interval $\Delta t$. The final particle 
position is in turn found by assuming that each particle maintains a constant relative position within a Lagrangian cell over the interval $\Delta t$. This particle position is expressed as a weighted sum of Lagrangian cell vertex coordinates at the end of the time step, where vertex coordinates are determined by calculating the intersection point of neighboring Lagrangian faces after the elapsed time $\Delta t$. To determine the final positions of Lagrangian faces, we assume that, during the current time step, each Lagrangian face has moved a total distance of $u_{f} \Delta t$ in the face outward normal direction. ${ }^{14}$

By replacing DSMC collision routines with the above procedures, we can approximate the motion of real atoms or molecules in a low-Kn gas flow over macroscopic length and time scales much larger than the mean free path and mean collision time respectively. This allows for cell sizes and $\Delta t$ values potentially several orders of magnitude larger than would be required in a DSMC simulation of the same flow. In fact, previous studies have demonstrated comparable accuracy to Euler or Navier-Stokes CFD calculations for low-Kn flow simulations employing the same grid. $^{14-16}$

\section{B. Hybrid Simulation Procedures}

In a hybrid LD-DSMC simulation, the LD method is used for low-Kn flowfield regions where DSMC is prohibitively expensive, while standard DSMC calculations are performed in high-Kn regions where continuum methods are inaccurate. Strong two-way coupling between LD and DSMC calculations is easily provided by allowing particles to move across the boundary between LD and DSMC domains. This boundary is determined dynamically during a simulation, by comparing a nondimensional continuum breakdown parameter to a userspecified cutoff value. The breakdown parameter evaluation is performed for all cells once every several hundred time steps, and a simple smoothing technique is used to avoid assignment of overly small regions to either LD or DSMC domains. ${ }^{15}$

These procedures can be implemented in an existing DSMC code far more quickly and easily than hybrid CFDDSMC methods, and the resulting algorithm is capable of accurately and efficiently simulating a wide range of multiscale flow problems for which a strongly coupled CFD-DSMC scheme would otherwise be required. The main limitation of the hybrid LD-DSMC algorithm is in application to flows involving a gas mixture; no consideration has previously been made for phenomena associated with mixtures. In particular, transport coefficients are determined in LD method procedures for a simple gas, and effects of mass diffusion are neglected.

\section{Transport Coefficients for a Mixture}

To extend the LD method for hybrid LD-DSMC simulation of flows involving a gas mixture, Wilke's mixing rule ${ }^{17}$ is employed in calculating the mixture dynamic viscosity $\mu_{\text {cell }}$ and thermal conductivity $k_{\text {cell }}$ in each cell within the LD domain during each time step. Cell-based values $\mu_{\text {cell }}$ and $k_{\text {cell }}$ are in turn found as functions of the viscosity $\mu_{s}$ and thermal conductivity $k_{s}$ for each species, which are computed using expressions based on the variable soft sphere (VSS) collision model of Koura and Matsumoto. ${ }^{18}$ As with the VHS model of Bird, ${ }^{1}$ the VSS model is commonly used in DSMC simulations to calculate binary collision cross sections, and employs an approximation that in a near-equilibrium flow the viscosity is proportional to the temperature raised to some constant power $\omega$. The VSS model is often used in place of VHS in DSMC simulations of mixture flows, due to its use of an additional constant $\alpha$ which allows for more accurate diffusive mass transport by means of anisotropic scattering in DSMC collisions. Thus, for better consideration of mass diffusion effects, the VSS model should be used in DSMC calculations as part of a hybrid LD-DSMC simulation of a gas mixture flow, and for consistency VSS should also be used in LD method calculations during a hybrid simulation.

\section{A. Species Transport Coefficients}

Using the VSS model, we can calculate the viscosity $\mu_{s}$ for some species $s$ in a given cell as

$$
\mu_{s}=\mu_{\text {ref,s }}\left(T_{\text {cell }} / T_{\text {ref }}\right)^{\omega_{s}}
$$

where $T_{\text {cell }}$ is the cell-averaged temperature during the current time step, $T_{\text {ref }}$ is a reference temperature, $\omega_{s}$ is a species-specific constant used in both VSS and VHS models, and $\mu_{\text {ref,s }}$ is the species viscosity at the reference temperature. The reference viscosity is calculated for each species at simulation startup, as a function of the mass per molecule $m_{s}$, species constants $\omega_{s}$ and $\alpha_{s}$, and a VSS collision diameter $d_{r e f, s}$ at the reference temperature $T_{\text {ref }}$ : 


$$
\mu_{r e f, s}=\frac{5\left(\alpha_{s}+1\right)\left(\alpha_{s}+2\right) \sqrt{m_{s} k_{B} T_{r e f} / \pi}}{4 \alpha_{s}\left(5-2 \omega_{s}\right)\left(7-2 \omega_{s}\right) d_{r e f, s}^{2}}
$$

where $k_{B}$ is Boltzmann's constant. ${ }^{1}$ Note that in the VHS model $\alpha_{s}=1$ and the species reference diameter $d_{r e f, s}$ has a slightly different value than in the VSS model. Once $\mu_{s}$ is known for a given species, the thermal conductivity $k_{s}$ can be determined using Euken's relation. ${ }^{19}$ For a species with $\zeta_{s}$ internal (rotational and vibrational) degrees of freedom, $k_{s}$ is calculated as

$$
k_{s}=\left(\frac{15}{2}+\zeta_{s}\right) \frac{\mu_{s} k_{B}}{2 m_{s}}
$$

\section{B. Mixture Properties}

Wilke's semi-empirical mixing rule ${ }^{17}$ is used to find cell-based mixture transport coefficients $\mu_{\text {cell }}$ and $k_{\text {cell }}$ once $\mu_{s}$ and $k_{s}$ have been computed for all species in the cell. Mixture transport coefficients are evaluated as weighted sums over the species values:

$$
\begin{aligned}
\mu_{\text {cell }} & =\sum_{s} N_{s} \mu_{s} / \phi_{s} \\
k_{\text {cell }} & =\sum_{s} N_{s} k_{s} / \phi_{s}
\end{aligned}
$$

Here $N_{s}$ is the number of particles of species $s$ currently assigned to the cell, and the parameter $\phi_{s}$ is evaluated through an additional summation over all species $r$ :

$$
\phi_{s}=\sum_{r} N_{r}\left[1+\sqrt{\frac{\mu_{s}}{\mu_{r}}}\left(\frac{m_{r}}{m_{s}}\right)^{1 / 4}\right]^{2} / \sqrt{8\left(1+\frac{m_{s}}{m_{r}}\right)}
$$

As mentioned above, accurate simulation of flows involving a gas mixture may require that diffusive mass transport be considered. Procedures for inclusion of diffusive mass transport in the LD method, as outlined below, involve the use of a mixture diffusion coefficient $D_{\text {cell }}$ which is updated during each time step and stored in the cell data structure along with $\mu_{\text {cell }}$ and $k_{\text {cell. }}$. By analogy with Eqs. (4) and (5), $D_{\text {cell }}$ can be computed as a function of the VSS self-diffusion coefficient for each species

$$
D_{\text {cell }}=\frac{1}{\rho_{\text {cell }}} \sum_{s} \frac{N_{s}}{\phi_{s}}\left[\frac{3 \alpha_{s}}{5}\left(\frac{7-2 \omega_{s}}{2+\alpha_{s}}\right) \mu_{s}\right]
$$

where $\rho_{\text {cell }}$ is the cell-based density associated with all particles currently assigned to the cell.

In a hybrid LD-DSMC simulation, values of $\mu_{\text {cell }}, k_{\text {cell }}$ and $D_{\text {cell }}$ are found using Eqs. (1) through (7) for all cells within the LD domain, during evaluation of cell-based quantities as described in Section II.

\section{Inclusion of Mass Diffusion Effects in the LD Method}

As described in a previous paper, ${ }^{16}$ diffusive momentum and energy transport can be modeled in the LD particle method by modifying procedures used to update cell-based temperature and bulk velocity values. These values are then assigned to all particles in a cell, after which particles are moved through the grid in a manner consistent with mass transport over macroscopic length scales in an inviscid flow. To account for mass transport due to molecular diffusion in a viscous flow, the cell-based approach used for diffusive momentum and energy transport cannot be employed, and an alternate approach - involving adjustments to particle trajectories - is required. Velocities used for particle movement should be modified in the presence of concentration gradients, so that particles of a given 
species tend to move in the direction of reduced species concentrations. This is accomplished through the use of species-specific Lagrangian cell face velocities, as outlined in the following discussion.

In the LD method procedures discussed in Section II, velocities used for particle movement are updated during each time step by forcing each particle to maintain a constant relative position over the time step interval $\Delta t$ with respect to a Lagrangian cell. ${ }^{14}$ Initial particle positions $\boldsymbol{x}_{\text {init }}$ at the beginning of the time step are expressed as a weighted sum of initial Lagrangian cell vertex coordinates, and the same weight coefficients are used with final vertex coordinates after time $\Delta t$ to find desired particle positions $\boldsymbol{x}_{\text {final }}$ at the end of the time step. Particle velocities are then set to equal $\left(\boldsymbol{x}_{\text {final }}-\boldsymbol{x}_{\text {init }}\right) / \Delta t$. The final Lagrangian cell vertex locations, which are used to find $\boldsymbol{x}_{\text {final }}$, are determined by calculating the intersection point of neighboring Lagrangian faces at the end of the time step. Each Lagrangian face has in turn moved a distance $u_{f} \Delta t$ in the face normal direction over the elapsed time $\Delta t$.

As a modification to these procedures for inclusion of mass diffusion effects, final particle locations $\boldsymbol{x}_{\text {final }}$ are evaluated as weighted sums of species-specific Lagrangian vertex coordinates. These coordinates correspond to movement of each Lagrangian cell face over a distance $\left(u_{f}+u_{d f, s}\right) \Delta t$ (instead of $u_{f} \Delta t$ as used previously) where $u_{d f, s}$ is the Fick's law diffusion velocity for species $s$ at the face $f$. Values of $u_{d f, s}$ are computed during every time step, for all simulated species at each interior cell face used in LD method calculations, using the expression

$$
u_{d f, s}=-\left.\frac{D_{f}}{Y_{f, s}} \frac{\partial Y_{s}}{\partial n}\right|_{f}
$$

Here $D_{f}$ is the diffusion coefficient evaluated at the face $f, Y_{f, s}$ is the mass fraction of species $s$ at the face, and $\partial Y_{s} /\left.\partial n\right|_{f}$ is the gradient of $Y_{f, s}$ in the direction of the outward normal unit vector $\boldsymbol{n}_{f}$ at the face. The diffusion coefficient is calculated as the density-weighted average of diffusion coefficients for cells on either side of the face. If diffusion coefficients for the current cell and the neighboring cell bordering face $f$ are denoted as $D_{\text {cell }}$ and $D_{\text {neigh }}$ respectively, and corresponding cell-based density values are given by $\rho_{\text {cell }}$ and $\rho_{\text {neigh }}$, then the face value $D_{f}$ is computed as

$$
D_{f}=\frac{\rho_{\text {cell }} D_{\text {cell }}+\rho_{\text {neigh }} D_{\text {neigh }}}{\rho_{\text {cell }}+\rho_{\text {neigh }}}
$$

Note that, to avoid simulation errors, the same $D_{f}$ value must be used for all species at a given face. The use of separate diffusion coefficients for each species could, for example, result in nonphysical bulk gas motion in a quiescent gas with uniform state quantities and a concentration gradient. In Eq. (8) the mass fraction $Y_{f, s}$ is set equal to the unweighted average of mass fractions $Y_{s}$ for species $s$ in the cells on either side of the face, where the cellbased mass fraction $Y_{s}$ is given by

$$
Y_{s}=\frac{N_{s} m_{s}}{\sum_{r} N_{r} m_{r}}
$$

The gradient $\partial Y_{s} /\left.\partial n\right|_{f}$ in Eq. (8) is found by dividing the difference in $Y_{s}$ values between the two cells by the facenormal projection of the vector difference in cell center coordinates $\boldsymbol{x}_{c}$ :

$$
\left.\frac{\partial Y_{s}}{\partial n}\right|_{f}=\frac{\left(Y_{s}\right)_{\text {cell }}-\left(Y_{s}\right)_{\text {neigh }}}{\boldsymbol{n}_{f} \cdot\left[\left(\boldsymbol{x}_{c}\right)_{\text {cell }}-\left(\boldsymbol{x}_{c}\right)_{\text {neigh }}\right]}
$$

In modifications to the LD simulation procedures involving Eq. (8), the effect of mass diffusion on the motion of an individual particle is dependent on the relative position of that particle with respect to its assigned cell. Diffusive mass transport can therefore occur in multiple directions within the cell, and particle trajectories should effectively reproduce the large scale bulk motion of atoms or molecules in the presence of a concentration gradient. 


\section{Evaluation of the Mass Diffusion Model}

To evaluate the above modifications to the LD particle method, we consider a simple one dimensional unsteady test case involving self diffusion in a quiescent gas. A simulation is performed using the modified LD method, with stationary argon gas at a temperature of $300 \mathrm{~K}$ and a number density of $1.4 \times 10^{21} \mathrm{~m}^{-3}$. The simulation uses a $0.5 \mathrm{~m}$ grid, divided into 50 equally spaced cells over an $x$ coordinate range between -0.25 and $0.25 \mathrm{~m}$, and a time step interval $\Delta t$ of $10^{-5} \mathrm{~s}$. Note that the cell size $\Delta x$ is approximately 10 times larger than the mean free path, while $\Delta t$ is about 5 mean collision times. The numerical weight of simulated particles is set so that around 20 particles are assigned to each cell. At simulation startup, all particles located on the left half of the grid $(x<0)$ are tagged as species 1, and all particles located on the right half of the grid $(x>0)$ are tagged as species 2 . The resulting discontinuity in species concentrations at $x=0$ creates a mixing layer, which gradually expands outward in both directions through diffusive particle movement. The simulation is stopped after a total elapsed time of $0.01 \mathrm{~s}$, or $1000 \Delta t$, and the instantaneous mass fraction profile $Y_{1}$ for species 1 at this final time is plotted in Fig. 1.

Figure 1 shows the final $Y_{1}$ profile over the full simulation domain. The total CPU time for this simulation is about $0.2 \mathrm{~s}$ on a single $2.33 \mathrm{GHz}$ processor. As shown in Fig. 1, some noticeable scatter is found in the $Y_{1}$ profile. This scatter is due mainly to a discretization in mass transport associated with the use of a finite number of particles per cell. The level of scatter can therefore be reduced by decreasing the numerical weight of each particle, to increase the number of particles per cell. This dependence of scatter on the number of particles is demonstrated in results, also shown in Fig. 1, from an additional simulation with around 1000 particles per cell. This second simulation shows greatly reduced scatter relative to the first simulation, and requires roughly $11 \mathrm{~s}$ of CPU time on a single processor.

For comparison, a DSMC simulation and a finite difference calculation for the same flow problem are performed, and results from these additional calculations are also shown in Fig. 1. The finite difference calculation is a first order solution to the diffusion equation

$$
\frac{\partial Y_{1}}{\partial t}=D \frac{\partial^{2} Y_{1}}{\partial x^{2}}
$$

Checks have been performed to ensure that the cell size $\Delta x$ and time step $\Delta t$ used to solve Eq. (12) are sufficiently small to avoid dependence on either $\Delta x$ or $\Delta t$. The diffusion coefficient $D$ in Eq. (12) is computed using Eq. (7) with the same VSS parameters ${ }^{1}$ as those used in the LD method simulation. In the DSMC simulation, $\Delta x$ and $\Delta t$ values are set to equal approximately one mean free path and one-half the mean collision time respectively, for a total of 500 cells and 10,000 time steps. To provide a level of statistical scatter in the DSMC results comparable to that of the LD simulation with 1000 particles per cell, around 10,000 particles per cell are required in the DSMC simulation. This corresponds to an increase in the total number of particles by a factor of 100 , with an increase in the number of time steps by a factor of 10. As expected, the DSMC simulation is around three orders of magnitude more expensive than the LD simulation with 1000 particles per cell; the DSMC simulation requires a total CPU time of slightly over 10 hours on eight processors in the NYX cluster at the University of Michigan.

In comparing the mass fraction profiles in Fig. 1, we find a similar level of agreement between results from the second LD simulation (with 1000 particles per cell) and the finite difference calculation as between DSMC and finite difference results. The root-mean-squared (RMS) difference between LD and finite difference profiles is approximately 0.0065 , while a larger RMS difference of about 0.0080 is found between DSMC and finite difference profiles. The largest local disagreement in these profiles is found near $x=0.1 \mathrm{~m}$ where, relative to the finite difference result, $Y_{1}$ is overestimated by 0.012 in the LD simulation and underestimated by 0.011 in the DSMC simulation. This corresponds to a percent error of $11 \%$ for the LD simulation and $10 \%$ for the DSMC simulation at $x$ $=0.1 \mathrm{~m}$. Similar differences in $Y_{1}$ are also observed at $x=-0.1 \mathrm{~m}$, where absolute errors are 0.010 and 0.012 for the LD and DSMC simulation respectively, and relative errors are about $1.1 \%$ for both simulations. The comparable level of agreement for LD and DSMC results can be seen as evidence of acceptable accuracy in the proposed mass diffusion model for the LD method.

Although the particle-based treatment of diffusive mass transport in the LD method is considerably less efficient than a direct solution to governing equations such as Eq. (12), the LD method does permit far more efficient macroscopic tracking of molecular motion in a low-Kn flow than is possible using DSMC. Because $\Delta x$ and $\Delta t$ values are not limited by the mean free path and mean collision time respectively, the LD method allows for enormous efficiency gains relative to DSMC in simulations of low-Kn flows. The mass diffusion model presented here should allow these gains to be realized in simulations of gas mixtures involving concentration gradients, for ultimate application to multiscale mixture flow simulations using a hybrid LD-DSMC approach.

\section{7}

American Institute of Aeronautics and Astronautics Approved for public release; distribution unlimited. 
Note in the proposed mass diffusion model that no explicit consideration is made for the effect of mass diffusion on energy transport. This effect is represented in the Navier-Stokes energy equation for a gas mixture by an added term involving species concentration gradients. ${ }^{20}$ In an LD simulation, the effect on energy transport is in fact accounted for implicitly, as follows: When particles are transported between neighboring cells in the presence of a concentration gradient, bulk kinetic and thermal energy assigned to each particle is transported as well, and some additional energy transport is associated with diffusive particle motion in the direction of the gradient.

Particle movement associated with diffusive mass transport between neighboring cells may also tend to increase diffusive momentum transport across cell faces, which can potentially increase artificial viscosity and reduce simulation accuracy in boundary layers or shear layers involving large concentration gradients. While any resulting loss of accuracy in the simulation will likely be very small, this effect should be acknowledged as a possible error source in some LD method calculations.

\section{Simulation of High Altitude Rocket Exhaust Flows}

As mentioned in the introduction, a strongly coupled hybrid LD-DSMC scheme has been developed for simulation of multiscale compressible flows involved a wide range of $\mathrm{Kn}$ regimes. The scheme has been implemented in the DSMC code MONACO ${ }^{21}$ and a series of detailed evaluations have been performed for a variety of test cases. Various models in this scheme have been tested through a series of comparisons with theoretical, numerical and experimental data, and models for viscous transport and wall slip boundary conditions have been individually verified. ${ }^{14-16}$ One ultimate goal in development of the hybrid scheme is unified simulation of the exhaust and atmospheric flowfield surrounding an upper stage rocket operating at high altitude, with a simulation domain which includes the entire nozzle and extends far downstream into the plume. Thus, to demonstrate capabilities of the code, we consider the flow around a generic sounding rocket at an altitude of $100 \mathrm{~km}$. While no existing numerical or experimental data for this case are available for comparison, previous evaluations of all components of the hybrid LD-DSMC algorithm provide confidence in overall simulation accuracy.

\section{A. Simulation Setup and Input Parameters}

An axisymmetric hybrid LD-DSMC simulation is performed for a rocket with a single $13 \mathrm{kN}$ thrust engine, on a simulation domain which extends $30 \mathrm{~m}$ radially outward from the central axis, $8 \mathrm{~m}$ upstream of the upper stage nozzle exit plane and $75 \mathrm{~m}$ downstream. This case is roughly based on recent stage separation simulations for a generic sounding rocket by Eramya et al. ${ }^{22}$ The nozzle has a convergence angle of $45^{\circ}$, a divergence angle of $15^{\circ}$, a curvature radius of $7 \mathrm{~cm}$ at the throat, and inlet, throat and exit diameters of $35 \mathrm{~cm}, 7 \mathrm{~cm}$ and $50 \mathrm{~cm}$ respectively. The outer surface of the rocket is modeled as a blunted cone/cylinder of total length $3 \mathrm{~m}$ and diameter $75 \mathrm{~cm}$, with a $2 \mathrm{~m}$ long cylindrical region and a nose radius of $5 \mathrm{~cm}$. The simulation boundary geometry is shown in Fig. 2 .

Ambient atmospheric conditions include a freestream velocity of $5 \mathrm{~km} / \mathrm{s}$, a temperature of $195.1 \mathrm{~K}$ and a total number density of $1.189 \times 10^{19} \mathrm{~m}^{-3}$. (These conditions are based on values at $100 \mathrm{~km}$ in the 1976 U.S. Standard

Atmosphere tables. ${ }^{23}$ ) The ambient atmosphere is modeled as a mixture of $\mathrm{N}_{2}$, with a mole fraction of 0.78 , and $\mathrm{O}_{2}$, with a mole fraction of 0.22 . Nozzle stagnation conditions include a pressure of $18.4 \mathrm{~atm}$ and a temperature of 2600 $\mathrm{K}$. Wall boundaries in both nozzle and external flow regions are given a temperature of $500 \mathrm{~K}$ and a thermal accommodation coefficient of 1.0. The exhaust flow consists of $\mathrm{CO}$ and $\mathrm{H}_{2} \mathrm{O}$, with mole fractions of 0.75 and 0.25 respectively at the inflow boundary along the nozzle inlet. The VSS collision model is used in both DSMC and LD calculations, and the newly proposed mass diffusion model as well as Wilke's mixing rule are employed in the LD method procedures. The total collision energy model of $\mathrm{Boyd}^{24}$ is used in DSMC procedures for nonequilibrium rotational excitation, and vibrational excitation is neglected in both DSMC and LD calculations.

The computational grid consists of about 110,000 triangular and quadrilateral cells, with a structured area in nozzle and nearfield plume regions and unstructured areas elsewhere in the grid. Cell dimensions are carefully limited to meet standard DSMC cell size guidelines in most atmospheric and farfield plume regions, while allowing for sufficient resolution in LD boundary layer calculations along the nozzle wall. DSMC guidelines are not met in some regions of expected low density far upstream and far downstream from the rocket, but gradients in these regions are generally very small and any resulting error is expected to be minimal. To further reduce errors associated with any overly large cells in the DSMC domain, a subcell technique ${ }^{1}$ is employed for selection of DSMC collision pairs, with a dynamically adapted subcell size of one-half the local mean free path. A close-up view of grid cells surrounding the rocket is shown in Fig. 3. 


\section{B. Techniques for Reducing Hybrid Simulation Expense}

Due to the complexity of the flowfield and the enormous range of densities, length scales and time scales involved, a number of techniques for improving simulation efficiency are required in order to avoid prohibitive computational expense. First, species-based numerical weighting is used to allow for sufficient particle populations in atmospheric flow regions, with numerical weight scaling factors of 1.0 for exhaust species $\mathrm{CO}$ and $\mathrm{H}_{2} \mathrm{O}$, and 0.1 for atmospheric species $\mathrm{N}_{2}$ and $\mathrm{O}_{2}$. Smaller cell-based numerical weights are applied in cells very close to the central axis, to reduce any loss of accuracy due to small particle populations along the axis. To further allow for a more uniform number of particles per cell, an automatic time step adaptation procedure is employed. In this procedure, the time step interval in each cell is periodically adjusted so that, subject to a maximum local time step corresponding to a CFL number of 0.5 , each cell contains no more than 25 particles. This adjustment in local time step dramatically reduces overall computational expense by limiting the number of particles per cell, while minimizing random walk errors in conserved quantities.

To avoid development of unphysical recirculating regions within the nozzle, two separate regions are used for flowfield initialization at simulation startup. One region, which includes only the convergent portion of the nozzle, is initialized with the subsonic nozzle inflow conditions. The second region, encompassing all remaining areas of the flowfield, is initialized with ambient atmospheric conditions. Both regions are assigned to the DSMC domain at startup, and a periodic (every 500 time steps) evaluation of continuum breakdown is used to assign continuum regions to the LD domain shortly afterward. Note that before the first evaluation of continuum breakdown, DSMC calculations are performed in the high density convergent nozzle region where DSMC cell size and time step guidelines are violated. While some resulting error is expected in transient flow properties early in the simulation, this error is found to have no effect on flow properties following convergence to steady state. Steady state conditions are assumed after roughly twice the number of time steps required for the total number of particles in the simulation to level off. Once steady state has been reached, cell-based sampling is performed to generate time-averaged results over a large number of time steps. For this simulation, sampling is initiated after 40,000 time steps, and the simulation is stopped after a total of 100,000 time steps.

After the simulation has completed, a post-processing utility is used to generate a new set of cell-based numerical weight assignments for use in a subsequent simulation. This utility reads in the time-averaged number of particles per cell at steady state, and assigns new weight values which satisfy the following criteria: At steady state each cell should have an average of between 20 and 40 particles, and neighboring cells with different numerical weights must have weight values which differ by a factor of two. This effectively creates bands of constant numerical weight across the simulation domain, and minimizes the frequency of particle cloning and removal while allowing for sufficient - but not overly large - particle populations in all cells. In a second simulation, these updated numerical weights are used along with cell-based time step values employed at steady state in the first simulation. (While numerical weight values could be optimized dynamically during the transient period of the first simulation, thus avoiding the need for a second simulation, the two step approach described here minimizes problems associated with statistical scatter in the time-averaged number of particles per cell. Such problems can distort bands of constant numerical weight, and can lead to an unnecessarily large number of particle cloning and removal operations with an associated loss of simulation accuracy.) Through a combination of nonuniform flowfield initialization, species-based weight scaling factors, variable numerical weights and variable time step intervals, this two step simulation approach is found to provide relatively high computational efficiency while avoiding any loss of accuracy associated with an insufficient number of particles in any cell.

Simulations are performed on 16 AMD Opteron processors in the NYX cluster at the University of Michigan. The first simulation, used for optimization of cell-based numerical weight assignments, requires roughly $130 \mathrm{CPU}$ hours, while the second simulation requires around $123 \mathrm{CPU}$ hours. In the second simulation, approximately 3.1 million particles are tracked through the grid at steady state, for an average of about 28 particles per cell. Results from the second simulation are presented in Figs. 4 through 12.

\section{Simulation Results}

Figure 4 shows flowfield domains used for DSMC and LD calculations at steady state. As displayed in the figure, the LD domain includes internal flow regions within the nozzle as well as a nearfield plume region which extends some distance downstream of the nozzle exit. Approximately $82 \%$ of cells are assigned to the DSMC domain, and the remaining 18\% are assigned to the LD domain. Two narrow areas (each two cells thick) along the continuum breakdown surface between DSMC and LD domains represent buffer regions, which are used for information transfer between DSMC and LD calculations. ${ }^{15}$

Figure 5 shows a close-up view of streamlines surrounding the rocket and within the nozzle. The dividing line between atmospheric and exhaust regions is clearly visible where streamlines from the two regions converge near

\section{9}

American Institute of Aeronautics and Astronautics Approved for public release; distribution unlimited. 
the nozzle exit. This line is pushed outward over the nozzle lip by the highly underexpanded nozzle flow, and an area of rapid expansion is shown in the plume region near the lip. As expected, streamlines within the nozzle converge through the subsonic expansion region upstream of the throat, then diverge downstream in a supersonic expansion. No clearly defined bow shock is shown near the nose, and the small curvature of streamlines over the rocket indicates a very weak compression wave. This may be explained by the high level of rarefaction in flowfield regions upstream of the nozzle, where the ambient mean free path is roughly $10 \mathrm{~cm}$ and the Knudsen number based on the rocket diameter is around 0.13 .

In Fig. 6, gas streamlines are shown over the entire simulated flowfield. As expected in a high altitude plume flow, an "inviscid core" expansion region appears directly downstream of the nozzle, with no well defined Mach disk or shock reflection features as typically found at lower altitudes. ${ }^{25}$ A distinct mixing layer - where atmospheric and exhaust flow streamlines converge - is bounded on either side by oblique shocks. These shocks appear in the figure as sudden changes in streamline direction, and post-shock regions of increased density are represented by a reduction in distance between neighboring streamlines. From a comparison between Figs. 4, 5 and 6, we find that the DSMC domain encompasses all regions of atmospheric flow, so that mixing between atmospheric and exhaust species occurs outside of the LD domain where the newly proposed mass diffusion model can influence species mixing processes. This means that the LD mass diffusion model likely has little or no effect on results for this flow problem. An evaluation of the influence of mass diffusion modeling for this case is presented below.

A close-up view of gas density contours surrounding the rocket is shown in Fig. 7, and contours of gas density over the entire flowfield are shown in Fig. 8. In the legend for both figures, density values for each contour line are normalized by the freestream atmospheric density of $5.702 \times 10^{-7} \mathrm{~kg} / \mathrm{m}^{-3}$. (A normalized density of 1.2 is used for contour level 1 to avoid excessive scatter associated with setting a contour level equal to the freestream density.) Note in the legend that the density varies by nearly seven orders of magnitude between the nozzle inlet and farfield plume regions. This corresponds to a similar variation in the size of the mean free path and the mean collision time, for a difference in local length and time scales well out of range for practical application of most CFD or DSMCbased simulation approaches.

In Fig. 7 we find the same rapid expansion around the nozzle lip observed in Fig. 5, as well as highly diffuse compression and expansion regions in the rarefied atmospheric flow over the rocket. A narrow region of increased density along the nozzle wall is also shown in Fig. 7. This trend can be explained by the reduction in gas expansion associated with lower bulk velocities within the nozzle wall boundary layer. Along radial planes within the divergent nozzle region and some distance downstream of the nozzle throat, the location of maximum density is actually slightly offset from the wall. This is evident in the shape of contour lines 10 and 11 within the nozzle, and is likely caused by the density reduction associated with higher gas temperatures along the wall.

The high density farfield region surrounding the mixing layer is clearly observed in Fig. 8, with a thin region of increased density which indicates the post-shock compression of the exhaust flow along the mixing layer. Also in Fig. 8, a gradual density reduction with downstream distance is found in the farfield core region of the plume. Some contour lines within this region - particularly for contour levels 3 and 4 - are inclined at large angles at the central axis. This inclination is unphysical, as no gradients in the radial direction should exist along the axis. It should be noted however that this type of unphysical result at the axis is commonly found in axisymmetric expansion flow simulations using DSMC and other particle methods.

Contours of gas translational temperature are shown over a small area surrounding the rocket in Fig. 9, and over the entire simulation domain in Fig. 10. Very low translational temperatures are observed in Fig. 10 through much of the farfield plume core region. In contrast, the translational temperature within the nearfield portion of the mixing layer approaches $7500 \mathrm{~K}$, and the maximum translational temperature in the diffuse shock layer near the nose is $8770 \mathrm{~K}$. While such high translational temperatures would seem to imply that vibrational excitation may be important, these temperatures occur in highly rarefied regions where large rotational relaxation times should allow for low molecular internal energy levels. In fact, the maximum rotational temperature anywhere in the flowfield is only $3608 \mathrm{~K}$. This temperature is found near the location of maximum translational temperature within the mixing layer.

Large differences between translational and rotational temperatures are demonstrated in Fig. 11, which shows profiles of both temperatures along a radial plane $0.5 \mathrm{~m}$ downstream of the nozzle exit. The horizontal dashed line in the figure denotes the boundary between DSMC and LD domains. Within this plane the translational temperature reaches a local maximum of $6675 \mathrm{~K}$, while the maximum rotational temperature is $3334 \mathrm{~K}$. Translational-rotational equilibrium is reached in regions within about $1 \mathrm{~m}$ of the axis, where the gas collision frequency is sufficiently high to prevent any significant rotational temperature lag. The temperature profile within these regions has a relatively complex shape, with a minimum translational/rotational temperature of $116 \mathrm{~K}$ in the rapid expansion region near the nozzle lip, and with additional features related to lip geometry and boundary layer formation along the nozzle wall.

10

American Institute of Aeronautics and Astronautics Approved for public release; distribution unlimited. 
Number density profiles for each species along the same radial plane are shown in Fig. 12. Here the mixing layer is found roughly $1 \mathrm{~m}$ from the axis; a sharp drop-off in number densities for atmospheric species is observed around this location, and a more gradual drop-off in exhaust flow species is shown further radially outward. The boundary between atmospheric and exhaust flow streamlines occurs approximately where species number density profiles cross, at a radial coordinate $r$ of $1.2 \mathrm{~m}$. The sudden increase in exhaust species number densities around $r=1 \mathrm{~m}$, and the less abrupt increase for atmospheric species between $r=1.8 \mathrm{~m}$ and $r=1.2 \mathrm{~m}$, can be attributed to post-shock density increases as mentioned in the above discussion of Figs. 6 and 8. Note that the more gradual density increase within the atmospheric flow region is a result of the high level of rarefaction in this region.

\section{Evaluation of Mass Diffusion Effects}

As discussed above, the entire LD domain shown in Fig. 4 lies within exhaust flow regions, and the plume/atmosphere mixing layer is completely inside the DSMC domain. It can therefore be assumed that any influence of the LD mass diffusion model is very small, and that simulation results are not significantly affected by inclusion of this model. To quantity any affects of this model for the rocket exhaust flow problem considered here, an additional simulation is performed with the LD mass diffusion model deactivated. In addition, to test the significance of mass diffusion in DSMC calculations, the VHS collision model is used in place of the VSS model, and species reference collision diameters $d_{r e f, s}$ are modified, as given by Bird, ${ }^{1}$ to avoid any differences in viscosity, thermal conductivity or DSMC collision frequency. Note that diffusive mass transport still occurs in DSMC calculations employing the VHS model, but mass diffusion is not considered independently from other viscous effects and the resulting diffusion coefficients are generally inaccurate.

In Fig. 13, species number density profiles along the radial plane at $x=0.5 \mathrm{~m}$ are shown for this new simulation and for the initial simulation which uses the VSS collision model and LD mass diffusion procedures. Results from the new simulation are shown as symbols, while number density profiles from the initial simulation (identical to those in Fig. 12) appear as lines. Very little disagreement is found here between results from the two different simulations; noticeable differences appear only in the slope of exhaust species number density profiles within the atmospheric flow region inside the mixing layer, where trace concentrations exist for these species. We can therefore conclude that, for this flow problem, mass diffusion has a negligible impact on bulk flow properties, and effects of mass diffusion can be ignored with no significant impact on simulation results. The same conclusion has been reached in additional comparisons between the two simulations involving other flow properties of interest.

\section{E. Simulation of the Flowfield During Stage Separation}

As a modification to the $100 \mathrm{~km}$ rocket exhaust flow described above, we next consider the same flowfield during a stage separation event. Following simulations of Eramya et al., ${ }^{22}$ a non-firing lower stage of the rocket is placed along the axis $1 \mathrm{~m}$ downstream of the nozzle exit plane. The lower stage is represented by a cylinder of length $3 \mathrm{~m}$ and diameter $75 \mathrm{~cm}$, with full thermal accommodation at the same temperature of $500 \mathrm{~K}$ used along wall boundaries for the upper stage. To simplify the problem, and to allow for considerable reduction in simulation expense through the use of variable cell-based time step values, we consider this as a steady state flow, with a constant separation distance between upper and lower stages and zero relative velocity for the lower stage. Note that quasi-steady state approximations have been demonstrated to allow for sufficient accuracy in a similar flow problem, due to the relatively large time scales associated with unsteady phenomena. ${ }^{22}$ The same two step hybrid simulation procedure is used as in the other simulations described above, with identical inflow properties and input parameters.

In Fig. 14, streamlines and LD/DSMC domain boundaries are shown over a small $8.5 \mathrm{~m}$ long region surrounding the two stages. The domain for LD method calculations here includes all interior regions within the nozzle, as well as a relatively small portion of the plume surrounding the lower stage. As found in the previous single stage case, the LD domain in Fig. 14 appears entirely within exhaust flow regions where streamlines originate at the nozzle inlet, so the newly proposed LD mass diffusion model should have no significant impact on simulation results. In observing the shape of streamlines and the separation distance between neighboring streamlines, we find a number of flowfield structures: First, as in the previous case, a highly diffuse compression region forms within the atmospheric flow surrounding the upper stage. A mixing layer appears where the rarefied atmospheric flow meets exhaust species expanding around the nozzle lip, and this layer is pushed radially outward by a high density plume impingement shock layer which forms along the upstream surface of the lower stage. A small recirculation zone is found within this shock layer, and another recirculation zone appears in the low density wake region around the downstream edge of the lower stage. The upstream recirculation zone may be an anomalous structure, possibly associated with a carbuncle ${ }^{26}$ as is often found in hypersonic blunt body simulations using CFD schemes which like the LD particle method ${ }^{14}$ - can effectively resolve stationary contact discontinuities. 
A view of streamlines over the entire simulation domain is shown in Fig. 15. As in the single stage case, the dividing line between atmospheric and exhaust flow streamlines is bounded on either side by oblique shocks, and these shocks are observed to gradually diverge from each other with increasing distance from the upper stage. However, in contrast to the previous case, the mixing layer forms at a very large angle relative to the axis, and plume impingement effects at the lower stage surface create a much wider farfield plume region. These trends can be observed in a comparison between Fig. 15 and Fig. 6. While no Mach disk is evident within the highly rarefied farfield plume region in Fig. 15, the "barrel" shock in the exhaust flow region adjacent to the mixing layer curves radially inward to meet the axis near the outflow boundary at $x=75 \mathrm{~m}$. The increased curvature of this shock, relative to the corresponding shock shown in Fig. 6, may be attributed to the lower densities in the farfield plume core region associated with streamline divergence around the lower stage.

Contours of gas density, normalized by the freestream density $5.702 \times 10^{-7} \mathrm{~kg} / \mathrm{m}^{-3}$, are shown over a small area surrounding the two stages in Fig. 16, and over the entire simulated flowfield in Fig. 17. Similar trends are observed in both figures as in Figs. 14 and 15. A rapid drop in density is found within the strong expansion regions around the nozzle lip and the upstream edge of the lower stage, and an outward-curving plume impingement shock is observed in Fig. 16 to form along the lower stage surface. A contour line along the upstream surface of the lower stage, corresponding to contour level 11 , is distorted by temperature and velocity gradients within the recirculating region described in the discussion of Fig. 14. As mentioned above, this recirculation region may be a spurious structure associated with the carbuncle phenomenon. ${ }^{26}$ The complicated shape of contour lines within the farfield plume core region shown in Fig. 17 can be explained by the influence of multiple weak expansion and compression waves, which form as a result of interactions between the nearfield plume flow and the lower stage. In comparing Fig. 17 to Fig. 8, we find lower densities in Fig. 17 within the farfield plume core region, due to the effect of the lower stage in diverting exhaust streamlines near the axis. While no existing numerical or experimental data is available for use in evaluating simulation accuracy, it should be noted that good qualitative agreement is found with published results of Eramya et al. $^{22}$ for DSMC plume simulations involving a similar flow.

\section{Summary and Conclusions}

A strongly coupled hybrid simulation scheme, implemented through minor changes to an existing DSMC code, has been modified to simulate gas mixtures involving an arbitrary number of species. Procedures have been described for determination of transport coefficients in continuum flowfield regions, using the VSS collision model $^{18}$ along with Wilke's mixing rule. ${ }^{17}$ An outline of continuum flow simulation procedures using a recently developed low diffusion (LD) method has been presented, and this method has been extended to incorporate effects of diffusive mass transport. The proposed LD mass diffusion model has been evaluated through a comparison with DSMC and finite difference calculation results for a simple one dimensional diffusion problem, and LD simulation results show good overall accuracy and very low statistical scatter relative to DSMC.

These new modifications to the LD method have been implemented in a hybrid LD-DSMC code for simulating multiscale flows involving a wide range of Knudsen number regimes. With these modifications, the LD-DSMC algorithm has been used to simulate the exhaust and atmospheric flow around a generic sounding rocket at an altitude of $100 \mathrm{~km}$. The simulation domain encompasses large farfield plume and atmospheric flow regions, and extends within the convergent-divergent nozzle to the nozzle inlet. A series of techniques have been utilized in order to reduce computational expense while preserving simulation accuracy. These techniques include dynamic variation in cell-based time step scaling factors, spatial and species-based variation in numerical weighting factors for the simulated particles, separate high density and low density regions for flowfield initialization at simulation startup, and a two step simulation procedure to optimize numerical weighting factors in each cell.

A number of flowfield properties have been examined in the rocket flow simulation results, and the influence of mass diffusion effects in this flow has been assessed through comparison with an additional hybrid LD-DSMC simulation. In this second simulation, mass diffusion is neglected in LD calculations and included at improper rates in DSMC calculations by using the VHS model in place of the VSS model. ${ }^{1}$ Results show that diffusive mass transport has a negligible impact on bulk flow properties throughout the simulated flowfield. Another simulation has been performed to consider the effect of plume impingement on a lower stage during a stage separation event. Large differences in flowfield characteristics, within both nearfield and farfield regions, have been found due to the presence of the lower stage.

The hybrid LD-DSMC scheme presented here is proposed as a general solution method for compressible gas flows involving both continuum and rarefied regions. The accuracy of standard DSMC is maintained within rarefied regions, while continuum calculations are performed using a DSMC-based particle method which preserves the underlying physics of the Navier-Stokes equations while avoiding the considerable implementation difficulty 
associated with hybrid CFD-DSMC schemes. It should be emphasized that the LD particle method is not intended as a general substitute for Navier-Stokes based CFD methods, for comparably efficient simulations of fully continuum flows with no rarefaction effects. Modern CFD methods - particularly high order methods - should in general be significantly more efficient than the LD method described here. (In a previous study, large efficiency gains were demonstrated for the LD method, relative to a finite volume CFD algorithm for the Euler equations, for an inviscid flow problem involving complex shock interactions. ${ }^{14}$ However, this comparison did not involve optimization of the CFD code for the problem of interest.) Instead, the LD method is intended primarily as a simple extension of an existing DSMC code to low Kn regimes, where DSMC limitations on cell size and time step cannot reasonably be met. Implementation of a hybrid code for strongly coupled LD and DSMC calculations is relatively straightforward, and allows an existing DSMC code to be easily modified for simulating multiscale flow problems which involve a wide range of Kn regimes.

In a broad class of such multiscale flows, the rarefied portion of the flowfield is sufficiently large (as measured by the fraction of cells in rarefied regions) that DSMC calculations account for a large majority of the total computational expense in a hybrid simulation. For these types of flows, the efficiency of the method employed for continuum calculations will have little impact on overall simulation efficiency. The proposed LD-DSMC scheme is ideally suited for simulating such flows, due to its significant advantages in ease of implementation relative to hybrid CFD-DSMC schemes, the high level of overall accuracy as demonstrated in previous studies, and the comparable efficiency to CFD-DSMC schemes expected for these types of flows. The high altitude rocket exhaust flow considered here is one example of a flow problem for which the efficiency of the continuum method likely has little effect, as about $82 \%$ of cells are assigned to the DSMC domain at steady state, and DSMC calculations account for most of the total simulation expense. It can therefore be argued that a hybrid CFD-DSMC simulation of this flow, with strong two-way coupling, consideration of wall slip effects, similar models for diffusive transport, full parallelization and optimization of the CFD algorithm for relevant flow regimes, would result in similar levels of both accuracy and efficiency while requiring the use of a much larger and more complicated code.

\section{Acknowledgments}

Financial support for this work was provided by Spectral Sciences, Inc. through contract W9113M-06-C-0122, a Missile Defense Agency (MDA) Small Business Innovative Research (SBIR) Phase II award. The authors gratefully acknowledge Jason Cline and Matt Braunstein for their oversight of this work.

\section{References}

${ }^{1}$ Bird, G. A., Molecular Gas Dynamics and the Direct Simulation of Gas Flows, Clarendon Press, Oxford, 1994.

${ }^{2}$ Papp, J. L., Wilmoth, R. G., Chartrand, C. C., and Dash, S. M., "Simulation of High-Altitude Plume Flow Fields Using A Hybrid Continuum CFD/DSMC Approach," AIAA Paper 2006-4412, 2006.

${ }^{3}$ VanGilder, D. B., Chartrand, C. C., Papp, J. L., Wilmoth, R. G., and Sinha, N., "Computational Modeling of Nearfield to Farfield Plume Expansion," AIAA Paper 2007-5704, 2007.

${ }^{4}$ Gimelshein, S. F., Levin, D. A., and Alexeenko, A. A., "Modeling of Chemically Reacting Flows from a Side Jet at High Altitudes," Journal of Spacecraft and Rockets, Vol. 41m No. 4, 2004, pp. 582-591.

${ }^{5}$ Guobiao, C., Jianhua, Z., Hui, T., Aiguo, C., and Yanguang, Y., "Numerical and Experimental Study of Small Thruster Plume," AIAA Paper 2004-3697, 2004.

${ }^{6}$ Hueser, J. E., Melfi, L. T., Bird, G. A., and Brock, F. J., “Analysis of Large Solid Propellant Rocket Engine Exhaust Plumes Using the Direct Simulation Monte Carlo Method," AIAA Paper 84-0496, 1984.

${ }^{7}$ Kaplan, C. R., Liu, J., and Oran, E. S., "Parallel Hybrid Method for Subsonic Flows: Coupling and Load-Balancing Challenges," AIAA Paper 2006-992, 2006.

${ }^{8}$ Schwartzentruber, T. E., and Boyd, I. D., "A Modular Particle-Continuum Numerical Method for Hypersonic Nonequilibrium Gas Flows," Journal of Computational Physics, Vol. 225, 2007, pp. 1159-1174.

${ }^{9}$ Wu, J.-S., Lian, Y.-Y., Cheng, G., Koomullil, R., and Tseng, K.-C.,"Development and Verification of a Coupled DSMC-NS Scheme Using Unstructured Mesh,” Journal of Computational Physics, Vol. 219, 2006, pp. 579-607.

${ }^{10}$ Hash, D. B., and Hassan, H. A., "Assessment of Schemes for Coupling Monte Carlo and Navier-Stokes Solution Methods," Journal of Thermophysics and Heat Transfer, Vol. 10, No. 2, 1996, pp. 242-249.

${ }^{11}$ Roveda, R., Goldstein, D. B., and Varghese, P. L., "Hybrid Euler/Direct Simulation Monte Carlo Calculation of Unsteady Slit Flow," Journal of Spacecraft and Rockets, Vol. 37, No. 6, 2000, pp. 753-760.

${ }^{12}$ Wadsworth, D. C., and Erwin, D. A., "Two-Dimensional Hybrid Continuum/Particle Approach for Rarefied Flows," AIAA Paper 92-2975, 1992.

${ }^{13}$ Garcia, A. L., Bell, J. B., Crutchfield, W. Y., and Alder, B. J., "Adaptive Mesh and Algorithm Refinement Using Direct Simulation Monte Carlo,” Journal of Computational Physics, Vol. 154, 1999, pp. 134-155. 
${ }^{14}$ Burt, J. M., and Boyd, I. D., “A Low Diffusion Particle Method for Simulating Compressible Inviscid Flows,” Journal of Computational Physics, Vol. 227, 2008, pp. 4653-4670.

${ }^{15}$ Burt, J. M., and Boyd, I. D., "A Hybrid Particle Approach for Continuum and Rarefied Flow Simulation," Journal of Computational Physics, 2008, in press (doi:10.1016/j.jcp.2008.09.022).

${ }^{16}$ Burt, J. M., and Boyd, I. D., "Extension of a Multiscale Particle Scheme to Near-Equilibrium Viscous Flows," AIAA Paper 2008-3914, 2008.

${ }^{17}$ Wilke, C. R., “A Viscosity Equation for Gas Mixtures,” Journal of Chemical Physics, Vol. 18, No. 4, 1950, pp. 517-519.

${ }^{18}$ Koura, K., and Matsumoto, H., "Variable Soft Sphere Molecular Model for Air Species," Physics of Fluids A, Vol. 4, 1992, pp. 1083-1085.

${ }^{19}$ Vincenti, W. G., and Kruger, C. H., Introduction to Physical Gas Dynamics, Krieger Publishing Company, Malabar, Florida, 1986.

${ }^{20}$ Gnoffo, P. A., Gupta, R. N., and Shinn, J. L., "Conservation Equations and Physical Models for Hypersonic Air Flows in Thermal and Chemical Nonequilibrium," NASA Technical Paper 2867, 1989.

${ }^{21}$ Dietrich, S., and Boyd, I. D., "Scalar and Parallel Optimized Implementation of the Direct Simulation Monte Carlo Method," Journal of Computational Physics, Vol. 126, 1996, pp. 328-342.

${ }^{22}$ Eramya, A., Cline, J., Braunstein, M., and Gimelshein, S., "Transient Modeling of High-Altitude Rocket-Stage Separation," Journal of Spacecraft and Rockets, Vol. 45, No. 4, 2008, pp. 698-705.

${ }^{23}$ U.S. Standard Atmosphere, 1976, U.S. Government Printing Office, Washington, D.C., 1976.

${ }^{24}$ Boyd, I. D., "Analysis of Rotational Nonequilibrium in Standing Shock Waves of Nitrogen," AIAA Journal, Vol. 28, 1990, pp. 1997-1999.

${ }^{25}$ Simmons, F. S., Rocket Exhaust Plume Phenomenology, Aerospace Press, El Segundo, CA, 2000.

${ }^{26}$ Pandolfi, M., and D'Ambrosio, D., 'Numerical Instabilities in Upwind Methods: Analysis and Cures for the 'Carbuncle' Phenomenon," Journal of Computational Physics, Vol. 166, 2001, pp. 271-301.

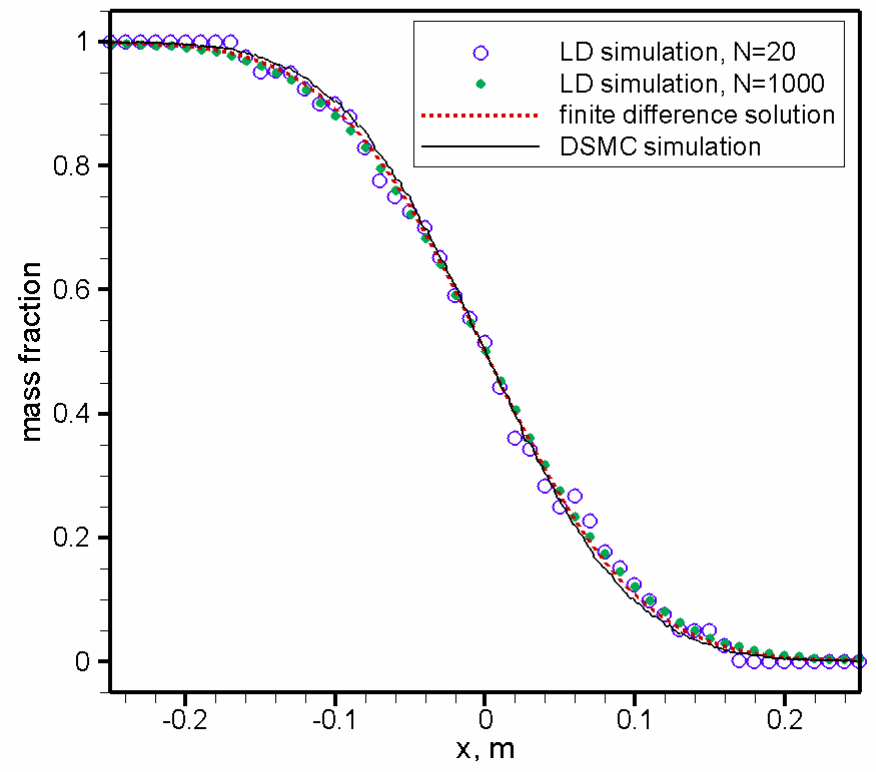

Figure 1. Mass fraction profiles at an elapsed time of $0.01 \mathrm{~s}$ for particles initially at $x<0$. 


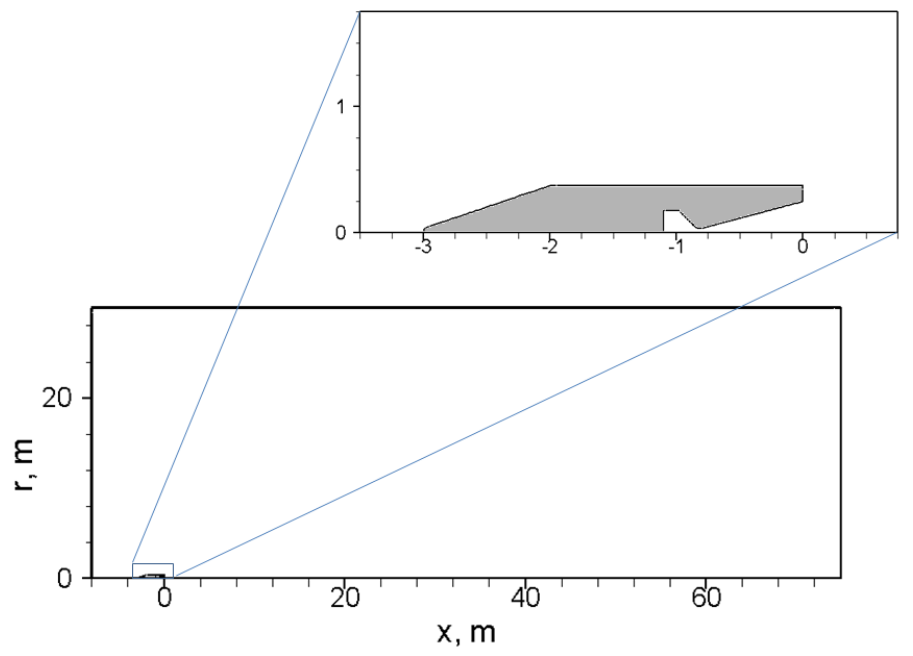

Figure 2. Boundary geometry for the rocket flow simulation.

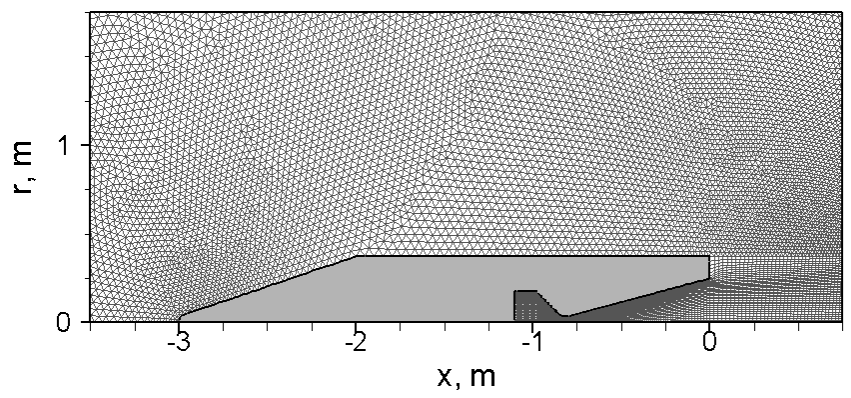

Figure 3. A close-up view of grid cells in a small region surrounding the rocket.

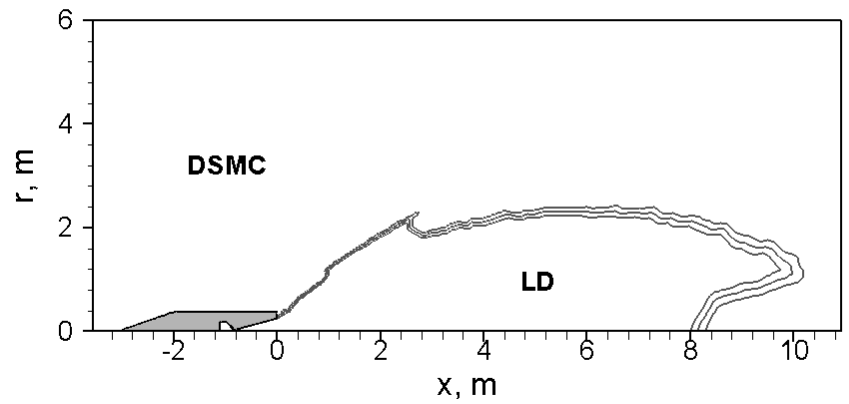

Figure 4. Domains for LD and DSMC calculations at steady state. Approved for public release; distribution unlimited. 


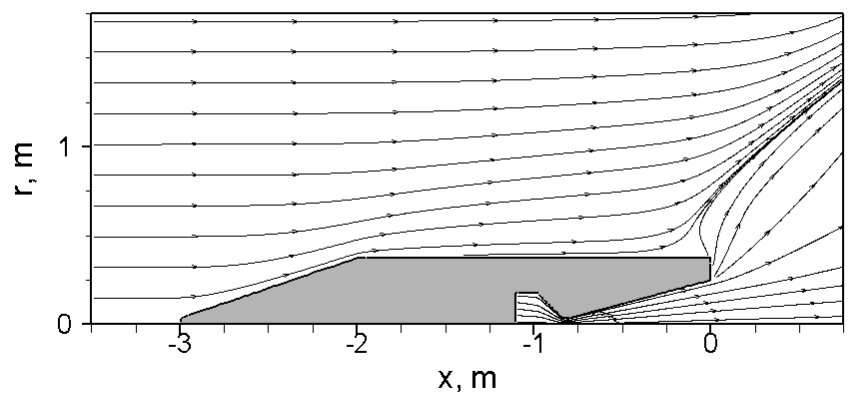

Figure 5. Streamlines in a small region surrounding the rocket.

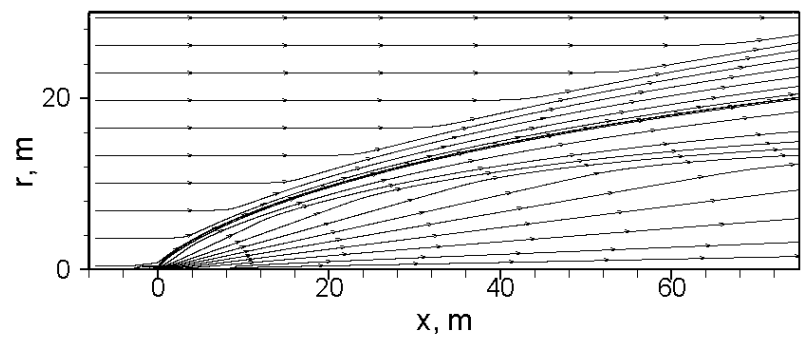

Figure 6. Streamlines over the full simulation domain.

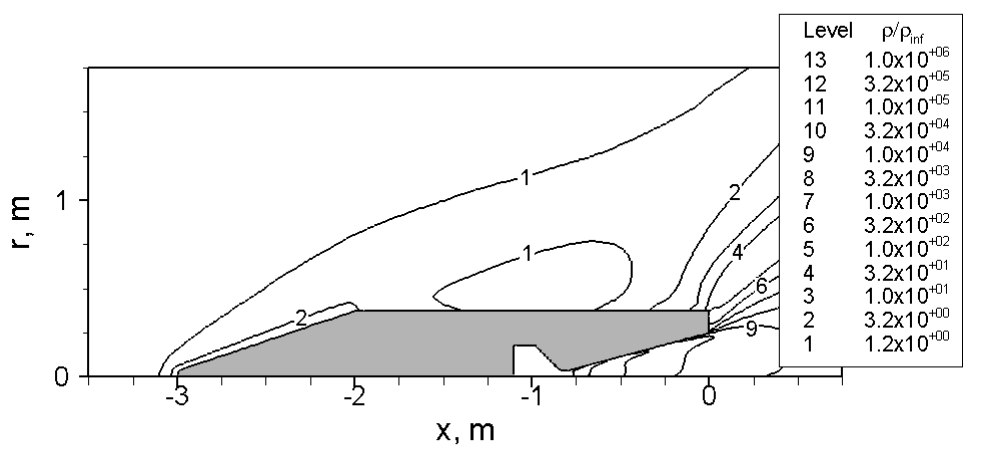

Figure 7. Contours of normalized density in a small region around the rocket. Approved for public release; distribution unlimited. 


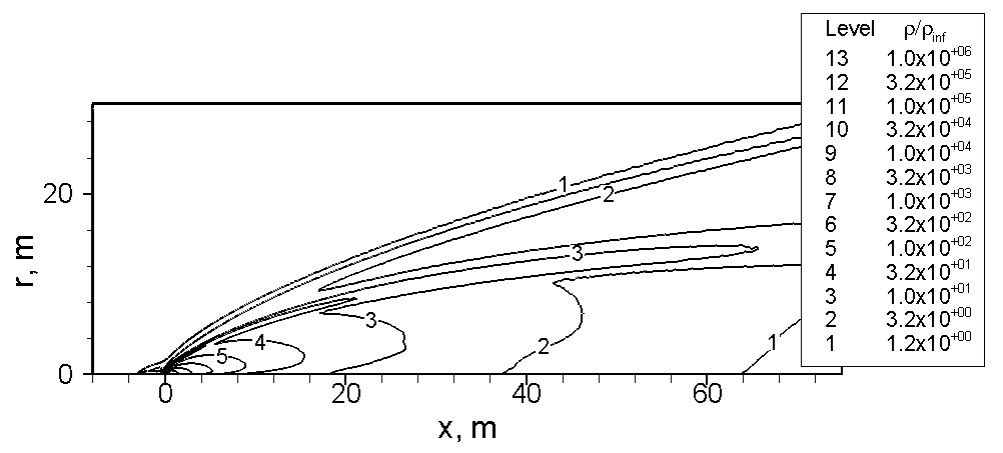

Figure 8. Normalized density contours over the entire flowfield.

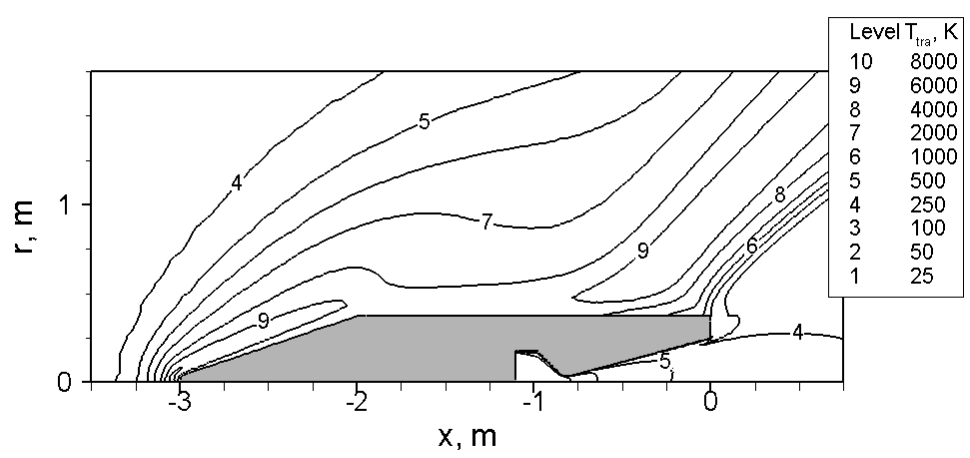

Figure 9. Contours of translational temperature in a small region around the rocket.

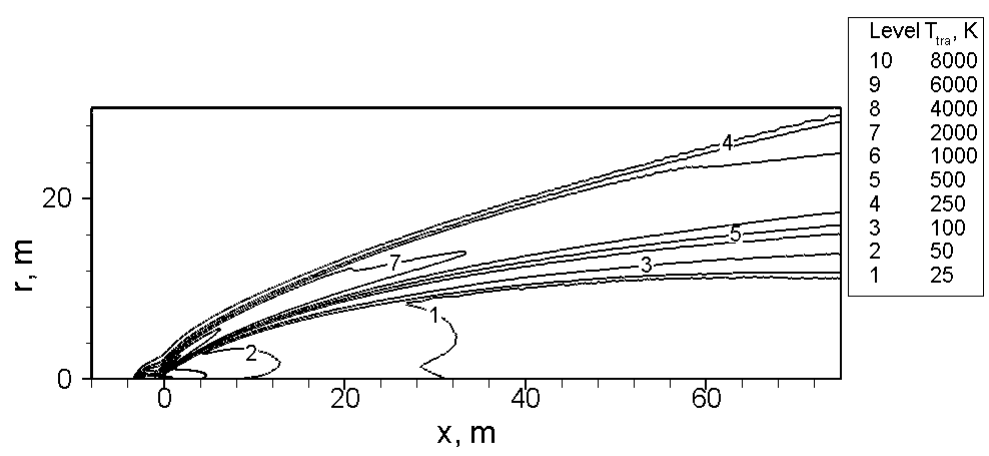

Figure 10. Contours of translational temperature over the entire flowfield. 


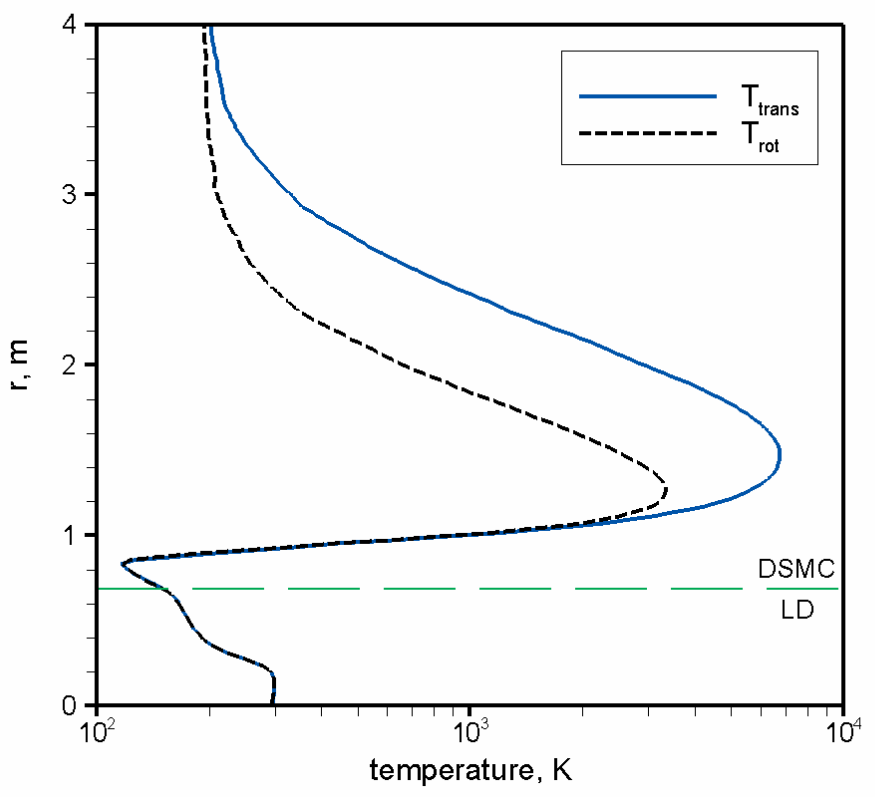

Figure 11. Profiles of translational and rotational temperature along a radial plane $0.5 \mathrm{~m}$ downstream of the nozzle exit.

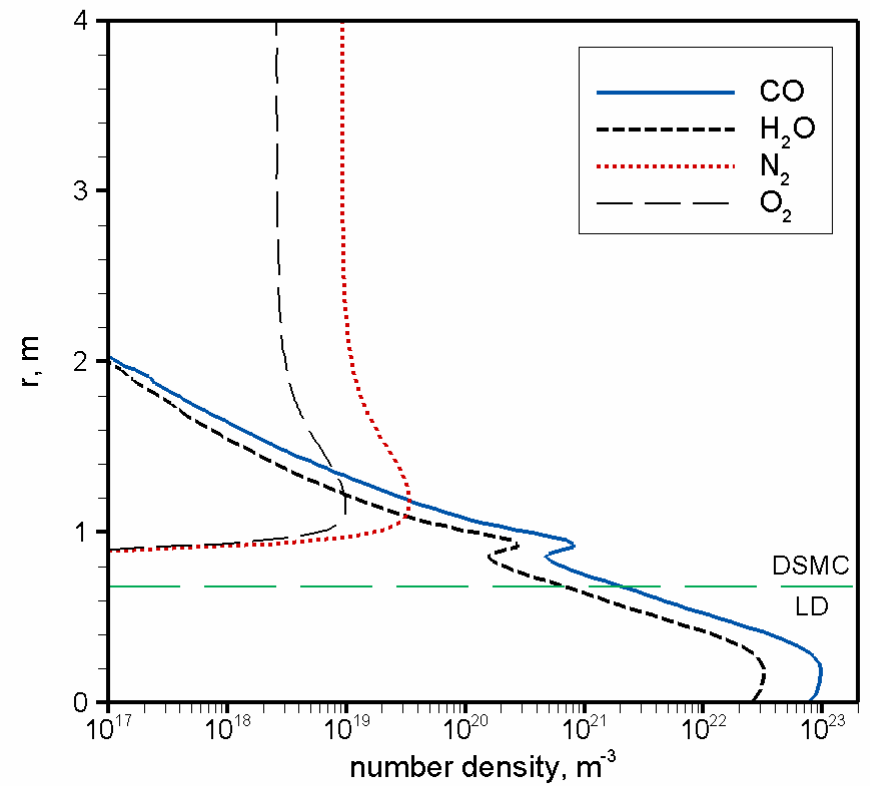

Figure 12. Profiles of species number density along a radial plane. 


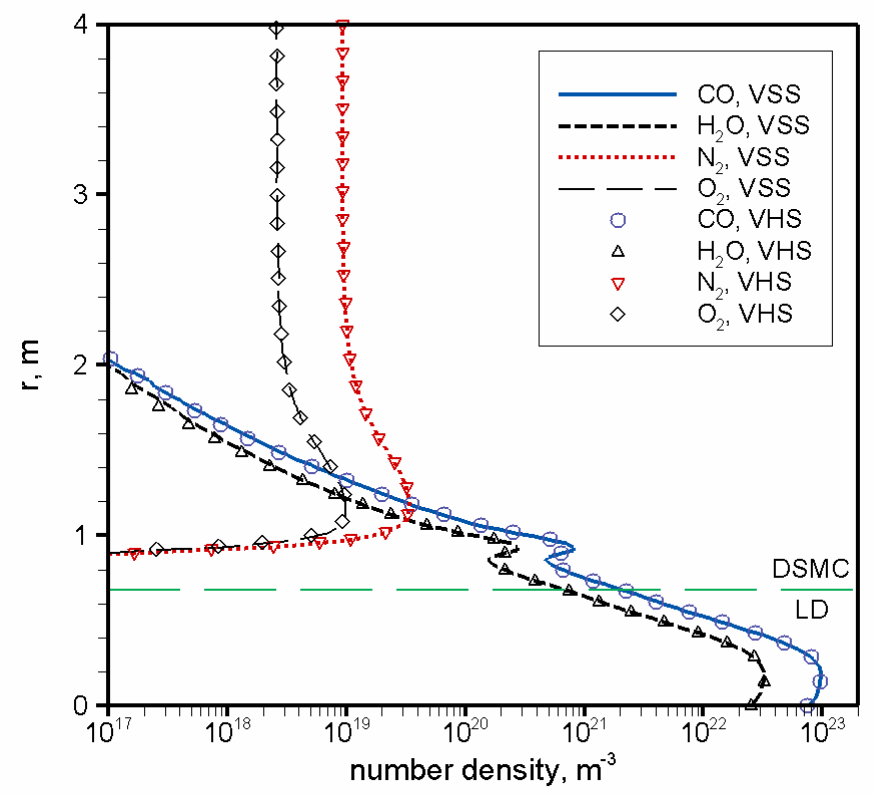

Figure 13. Comparison of species number density profiles with and without consideration of diffusive mass transport.

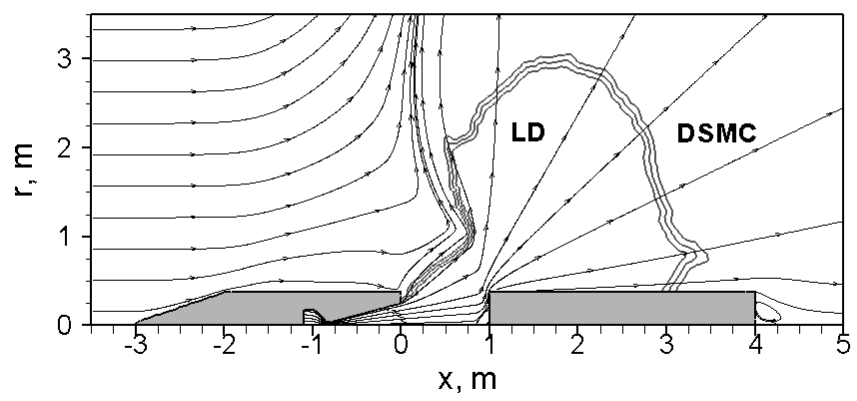

Figure 14. A close-up view of streamlines and LD/DSMC domain boundaries for a stage separation flow.

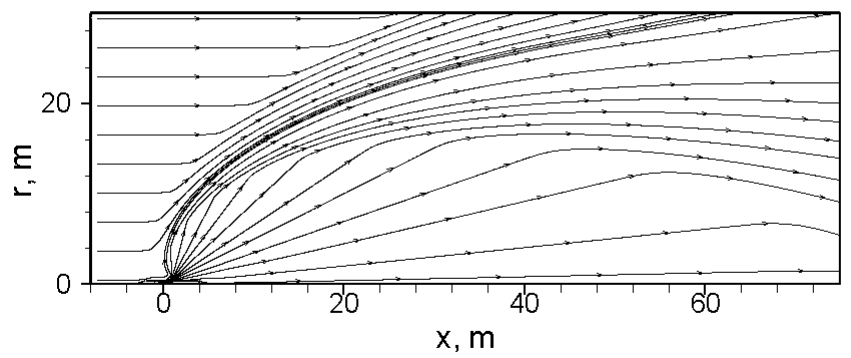

Figure 15. Streamlines for the stage separation flow over the entire simulation domain. Approved for public release; distribution unlimited. 


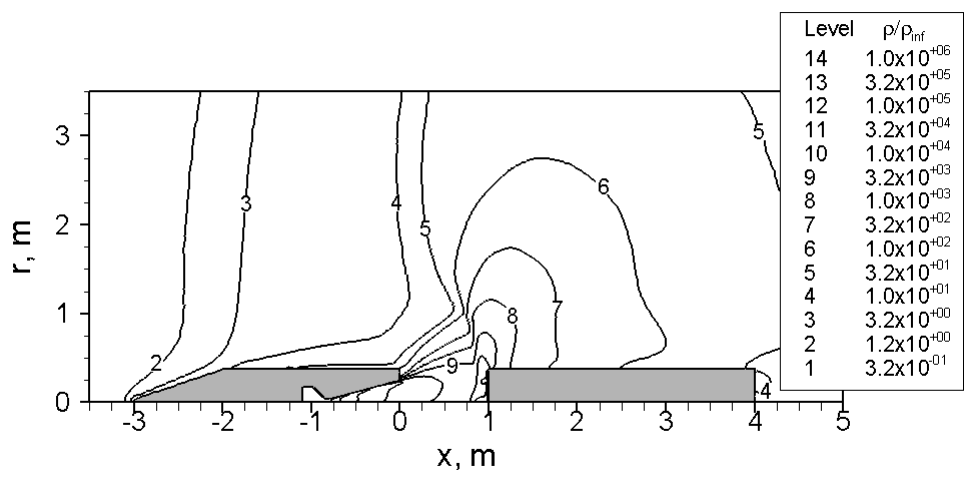

Figure 16. A close-up view of normalized density contours for the stage separation flow.

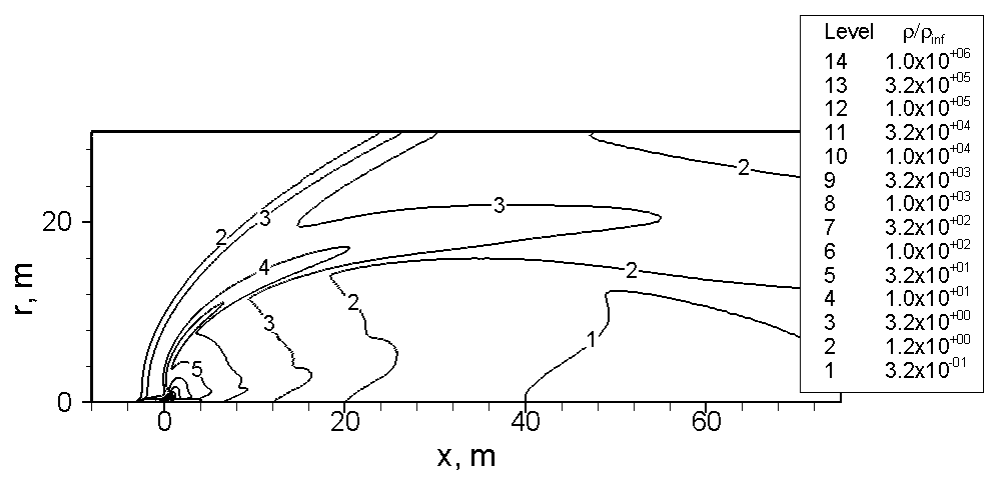

Figure 17. Normalized density contours for the stage separation flow over the entire simulation domain. 